\title{
IDENTIFICACIÓN DE PARÁMETROS MODALES DE ESTRUCTURAS VIBRANTES CON EXCITACIÓN ESTOCÁSTICA O DESCONOCIDA
}

\author{
Recepción: Noviembre de 2005 / Aceptación: Diciembre de 2005
}

(1) Roberto Amaro Baldeón

(2) Paulo Gardel Kurka

\section{RESUMEN}

El Modelo multivariado autoregresivo con medias móviles(VARMA) se emplea para identificar características dinámicas de un sistema estructural ante la presencia de ruido. Para estimar los parámetros del Modelo VARMA, se emplea el algoritmo de Spliid. Para determinar los parámetros modales, la matriz compañera se forma con los coeficientes de la parte autoregresiva del Modelo VARMA. La eficiencia de este modelo, discutida aquí, se analiza por medio de un sistema vibratorio de tres grados de libertad.

Palabras Clave: VARMA, análisis modal, matriz compañera, multivariado, estimación, mantenimento preventivo.

IDENTIFICATION OF MODAL PARAMETERS OF VIBRATING STRUCTURES WITH UNKNOWN OR STOCHASTIC EXCITATION

\section{ABSTRACT}

The Vector Autoregressive Moving Average (VARMA) Model is used to identify dynamical characteristics of a structural system in the presence of noise. In order to estimate the parameters of the VARMA Model, the Spliid's fast algorithm is used. To determine the modal parameters the companion matrix is built with the autoregressive part of the VARMA Model. The performance of this method here discussed is presented by means of simulations, using three degrees of freedom mass-dampingstiffness vibrating system.

Key words: VARMA, modal analysis, companion matrix, multivariate, estimation.

(1) Magíster en Ingeniería Industrial. Profesor del Departamento de Matemática, Universidade Estadual de Mato Grosso (Brasil) E-mail:amarobrp@fem.unicamp.br

(2) Profesor del Departamento de Projeto Mecânico PósGraduação, Faculdade de Engenharia Mecânica, Universidade Campinas (Brasil). E-mail:kurka@fem.unicamp.br

\section{INTRODUCCIÓN}

En el caso del análisis modal frecuentemente, los datos procesados se obtienen en condiciones de laboratorio. Sin embargo, en muchas aplicaciones industriales, los datos obtenidos son muy diferentes a los datos de laboratorio, lo que implica el desarrollo de modelos que permitan analizar datos en condiciones operacionales [1], lo que es muy común en el mantenimiento preventivo de maquinarias, ya que en este caso los datos de entrada no son medidos, sólo se dispone de datos de salida, por lo que sólo con los datos de salida se tiene que obtener información acerca de cómo la maquina esta vibrando y cuál es su amortecimiento para poder hacer un diagnóstico y una detección de falla [2].

Una técnica muy usada en análisis operacional es la técnica "Pick picking", aplicada a las funciones de densidad espectral cruzada [2]. Seleccionando los picos en la curva espectral, se pueden obtener estimaciones aproximadas de las frecuencias de resonancia y de formas de deflexión, luego éstas, comparadas con las que se obtienen en condiciones de laboratorio, permiten tomar decisiones, con respecto a que si se requiere el mantenimiento.

En el presente trabajo, se presenta el modelo Multivariado Autoregresivo con Medias Móviles (VARMA) para el análisis en condiciones operacionales. Los modelos VARMA se han analizado en extenso en la bibliografía [5, 7, $8,9,10]$ y han sido muy aplicados en situaciones donde sólo se disponen de datos de salida [2, 5, 7, 8, 9, 10]. Es conocido que la parte autoregresiva del modelo VARMA contiene las características del sistema mecánico analizado $[5,9]$. Por consiguiente, es necesario estimar los parámetros del modelo VARMA. Para tal estimación, se emplea mucho el algoritmo de Spliid [3,6]. La gran ventaja de este algoritmo es que, cuando se dispone de una gran cantidad de datos y las condiciones de regularidad son satisfechas, los resultados obtenidos se parecen mucho a los que se han obtenidos empleando técnicas de máxima verosimilitud [3, 6,11]. Por otro lado, para la obtención de los parámetros modales, la matriz compañera se construye con las estimaciones de los parámetros de la parte autoregresiva del modelo propuesto [5, 9]. La aplicabilidad de dicho modelo se hará simulando un sistema mecánico de tres grados de libertad.

\section{MODELO VARMA}

El modelo VARMA se expresa mediante la ecuación (1):

$$
Y_{t}-\sum_{i=1}^{p} \phi_{i} Y_{t-i}=\varepsilon_{t}-\sum_{i=1}^{q} \Theta_{i} \varepsilon_{t-i}
$$


donde:

$Y_{t}=\left(y_{1 t}, y_{2 t}, \ldots \ldots ., y_{k t}\right)^{T}, \quad \varepsilon_{t}=\left(\varepsilon_{1 t}, \varepsilon_{2 t}, \ldots \ldots ., \varepsilon_{k t}\right)^{T}$

son $\mathrm{k}$ vectores componentes, $\mathrm{y}$

$\phi_{1}, \phi_{2}, \ldots . ., \phi_{p}, \Theta_{1}, \Theta_{2}, \ldots ., \Theta_{q}$ representan matrices de parámetros no conocidos.

Es sabido que con las raíces del polinomio característico, formado con las estimaciones de los parámetros de la parte autoregresiva del modelo VARMA, pueden obtenerse las características dinámicas del sistema mecánico.

Algoritmo para la Estimación de los Parámetros del Modelo VARMA

El algoritmo usado para la estimación de los parámetros del modelo VARMA fue desarrollado por Spliid $(3,6)$.

Sea la matriz de observaciones en el tiempo, cuya dimensión es $\mathrm{k} \times \mathrm{N}$ :

$$
y=\left[\begin{array}{ccc}
y_{11} & \cdots & y_{1 N} \\
\vdots & \ddots & \\
y_{1 k} & \cdots & y_{k N}
\end{array}\right]=\left\{y_{i j}\right\}, i=1, . ., k \quad j=1, \ldots, N
$$

de manera análoga, se define la matriz de residuales

$$
\left\{\varepsilon_{i j}\right\}, i=1, . ., k \quad j=1, \ldots, N
$$

Por otro lado, se definen las matrices de rezagos:

$$
\begin{aligned}
Y & =\left(L y, L^{2} y, \ldots . ., L^{p} y\right) \\
A & =\left(L \varepsilon, L^{2} \varepsilon, \ldots . ., L^{p} \varepsilon\right) \\
U & =(-A, Y)
\end{aligned}
$$

donde $L^{s}=\left\{y_{i, j-s}\right\}, i=1, \ldots, k \quad j=1, \ldots, N$

Las matrices de los coeficientes son ordenadas en una única matriz de orden $(\mathrm{kq}+\mathrm{kp}) \times \mathrm{k}$ :

$$
\delta=\left(\theta_{1}, \theta_{2}, \ldots, \theta_{q}, \phi_{1}, \phi_{2}, \ldots, \phi_{p}\right)^{T}
$$

El algoritmo se inicia sin parte de medias móviles pero, en compensación el número de términos autoregresivos, se incrementa, $s$ (en la práctica $s=p$ $+q)$. A continuación, se presenta el mencionado algoritmo. Es importante acotar que este algoritmo no necesita valores iniciales.

Procedimiento

Paso 0: Construir la matriz $W=\left(L y, L^{2} y, \ldots \ldots \ldots, L^{s} y\right)$, luego por regresión lineal se tiene:

$$
\hat{\varepsilon}(0)=y-W\left(W^{T} W\right)^{-1} W^{T} y
$$

donde $\hat{\varepsilon}(0)$ denota el valor estimado de la matriz $\varepsilon$ obtenido en la iteración 0 . Luego, se hace $\hat{\delta}(0)=0, j=0$ y se va para el paso 2.

Paso 1: Calcular recursivamente las estimaciones de los residuales para $t=1,2, \ldots \ldots, N$ :

$$
\hat{\varepsilon}_{t}(j)=y_{t}-\sum_{i=1}^{p} \hat{\phi}_{i}(j) y_{t-i}+\sum_{i=1}^{q} \hat{\theta}_{i}(j) \hat{\varepsilon}_{t-i}
$$

Paso 2: Construir $\hat{A}(j)$, formar la matriz $\hat{U}(j)$ y calcular las nuevas estimaciones de los parámetros, resolviendo la siguiente ecuación:

$$
\hat{U}^{T}(j) \hat{U}(j) \hat{\delta}(j+1)=\hat{U}^{T} y
$$

Paso 3: Si $\hat{\delta}(j+1) \neq \hat{\delta}(j)$, incrementar j en 1 y repetir los pasos 1 hasta 3 , si $\delta(j+1)=\hat{\delta}(j)$ parar.

Spliid $(3,6)$ muestra que los estimadores obtenidos, usando su algoritmo, tienen distribución asintóticamente normal. Otra ventaja de este algoritmo es que es bastante rápido, en comparación con los métodos de máxima verosimilitud.

\section{Orden del Modelo}

Pare escoger el orden del modelo VARMA, se emplea el Criterio Bayesiano de Schwarz (BCS) [4]:

$$
S B C=\log (\hat{\Sigma} \mid)+\frac{r \log (T)}{T}
$$

donde:

$r$ : es el número de parámetros estimados

$T$ : es el número de muestras

$\Sigma$ : es la matriz de covariancia

Estimación del Parámetro Modal (Modal Parameter Estimation)

Para obtener las características modales del sistema mecánico es necesario formar la matriz compañera $M[5,9]$,

$$
M=\left[\begin{array}{ccc}
-\phi_{1} & \cdots & -\phi_{p} \\
I_{(p-1)} & \multicolumn{2}{c}{0_{(p-1) x 1}}
\end{array}\right]
$$

Los autovalores y autovectores de $\mathrm{M}$ se relacionan con los parámetros modales $[5,12,13]$ de la siguiente forma:

$$
\lambda_{s}=\frac{\ln \left(\lambda_{M}\right)}{d t} \quad \psi_{M}=\left[\begin{array}{c}
\psi_{S} \\
\cdots \\
\psi_{2}
\end{array}\right]
$$

donde:

$\lambda_{M}$ : representa los autovalores de $\mathrm{M}$

$\lambda_{s}$ : representa los autovalores del sistema mecánico

$\psi_{M}$ : representa los autovectores de M 
Cuadro 1. Comparación entre frecuencias y amortecimientos naturales teóricos con frecuencias y amortecimientos naturales estimados sin ruido

\begin{tabular}{|c|c|c|c|}
\hline $\begin{array}{c}\text { Frecuencias } \\
\text { Naturales } \\
\text { Teóricas }(\mathrm{Hz})\end{array}$ & $\begin{array}{c}\text { Frecuencias } \\
\text { Naturales } \\
\text { Estimadas }(\mathrm{Hz})\end{array}$ & $\begin{array}{c}\text { Amortecimiento } \\
\text { Teórico }\end{array}$ & $\begin{array}{c}\text { Amortecimiento } \\
\text { Estimado }\end{array}$ \\
\hline 0.6631 & 0.6617 & 0.0114 & 0.0144 \\
\hline 1.1010 & 1.1025 & 0.031 & 0.015 \\
\hline 2.5898 & 2.5909 & 0.080 & 0.084 \\
\hline
\end{tabular}

Cuadro 2. Comparación entre modos de vibrar teóricos y estimados

\begin{tabular}{|c|c|}
\hline $\begin{array}{c}\text { Modos de Vibrar } \\
\text { Teóricos }\end{array}$ & $\begin{array}{c}\text { Modos de Vibrar } \\
\text { Estimados }\end{array}$ \\
\hline 1.0000 & 1.0000 \\
$-0.0884+0.0008 \mathrm{i}$ & $-2.6122+0.0110 \mathrm{i}$ \\
$-0.0992+0.0010 \mathrm{i}$ & $-1.7189+0.0101 \mathrm{i}$ \\
\hline 1.0000 & 1.0000 \\
$6.5732+0.0404 \mathrm{i}$ & $0.2624+0.0026 \mathrm{i}$ \\
$4.2208+0.0250 \mathrm{i}$ & $0.1438+0.0017 \mathrm{i}$ \\
\hline 1.0000 & 1.0000 \\
$-15.4828-0.2628 \mathrm{i}$ & $-16.3137+3.4079 \mathrm{i}$ \\
$23.8749+0.4254 \mathrm{i}$ & $25.3193-5.2711 \mathrm{i}$ \\
\hline
\end{tabular}

$\psi_{s}$ : representa los autovectores del sistema mecáni$\mathrm{co}$, el cual es la mitad superior de la matriz $\psi_{M}$.

\section{RESULTADOS}

Para aplicar la teoría que se ha presentado hasta el momento, se simuló un sistema de tres grados con dos entradas y tres salidas. Para utilizar la metodología propuesta sólo utilizaremos los datos de salida. Se presentan dos simulaciones: En la primera, no se contaminan los datos de salida con ruido estocástico, mientras que, en el segundo caso, sí.

Caso 1: Simulación sin Contaminación en la Salida

Aplicando el modelo $\operatorname{VARMA}(2,2)$ se puede apreciar, en el Cuadro 1, que las frecuencias de vibración son bien aproximadas, mientras que los amortecimientos lo son en menor medida. Por otro lado, en el Cuadro 2, se presentan los modos de vibrar estimados. En este caso, sólo un modo de vibrar es bien aproximado, los otros mantienen sólo sus signos.

Caso 2: Simulación con Contaminación en la Salida

En el presente caso, se contaminó la salida con ruido estocástico que llevó a una razón ruido/salida de 0.30 . Aplicando el modelo $\operatorname{VARMA}(10,10)$, se pue-
Cuadro 3. Comparación entre frecuencias y amortecimientos naturales teóricos con frecuencias y amortecimientos naturales estimados sin ruido

\begin{tabular}{|c|c|c|c|}
\hline $\begin{array}{c}\text { Frecuencias } \\
\text { Naturales } \\
\text { Teóricas }(\mathrm{Hz})\end{array}$ & $\begin{array}{c}\text { Frecuencias } \\
\text { Naturales } \\
\text { Estimadas }(\mathrm{Hz})\end{array}$ & $\begin{array}{c}\text { Amortecimiento } \\
\text { Teórico }\end{array}$ & $\begin{array}{c}\text { Amortecimiento } \\
\text { Estimado }\end{array}$ \\
\hline 0.6631 & 0.6632 & 0.0114 & 0.0106 \\
\hline 1.1010 & 1.1003 & 0.031 & 0.017 \\
\hline 2.5861 & 2.5861 & 0.080 & 0.080 \\
\hline
\end{tabular}

Cuadro 4. Comparación entre modos de vibrar teóricos y estimados

\begin{tabular}{|c|c|}
\hline $\begin{array}{c}\text { Modos de Vibrar } \\
\text { Teóricos }\end{array}$ & $\begin{array}{c}\text { Modos de Vibrar } \\
\text { Estimados }\end{array}$ \\
\hline 1.0000 & 1.0000 \\
$-0.0884+0.0008 \mathrm{i}$ & $-2.6457+0.2280 \mathrm{i}$ \\
$-0.0992+0.0010 \mathrm{i}$ & $-1.6883+0.1536 \mathrm{i}$ \\
\hline 1.0000 & 1.0000 \\
$6.5732+0.0404 \mathrm{i}$ & $0.2653+0.0016 \mathrm{i}$ \\
$4.2208+0.0250 \mathrm{i}$ & $0.1378+0.0042 \mathrm{i}$ \\
\hline 1.0000 & 1.0000 \\
$-15.4828-0.2628 \mathrm{i}$ & $-9.3866+8.3737 \mathrm{i}$ \\
$23.8749+0.4254 \mathrm{i}$ & $12.9611-10.6791 \mathrm{i}$ \\
\hline
\end{tabular}

de apreciar, en el Cuadro 3, que las frecuencias de vibración son bien aproximadas, igual que en el caso sin ruido, mientras que los amortecimientos también tienen aproximaciones buenas, mejores que en el caso sin ruido. Por otro lado, en el Cuadro 4, se presentan los modos de vibrar estimados. En este caso, sólo un modo de vibrar es bien aproximado, los otros mantienen sólo sus signos, igual que en el caso sin ruido.

\section{CONCLUSIONES}

El modelo propuesto para la determinación de características dinámicas de un sistema mecánico, bajo la influencia de ruido aleatorio y con sólo datos de salida, da buenos resultados, en cuanto a las frecuencias de vibración y también da resultados relativamente buenos, con respecto a los amortecimientos. En general, el modelo VARMA, que se ha señalado, presenta buenas estimativas de las características dinámicas del sistema de tres grados de libertad.

\section{BIBLIOGRAFÍA}

1. Van Der Auweraer, H. (2001). Structural Dynamics Modeling using Modal Analysis: Applications, Trends and Challenges. IEEE Instrumentation and Measurement. Technology Conference. May 21-23. 
2. Hermans, L., Guillame, P. (1998). A frequencyDomain Maximum Likelihood Approach for the Extraction of Modal Parameters from output-only Data. Proceeding of ISMA 23. Volume 1.

3. Spliid, H. (1983). A Fast Estimation for the Vector Autoregressive Moving Average Models With Exogenous Variables. Journal of the American Statistical Association, 83 1097-1107.

4. SAS Institute. (2000). Manual VARMAX. Institute Inc, NC, USA.

5. Larbi, N. y Lardies, J. (2000). Experimental Modal Analysis of Structure Excited by a Random Force. Mechanical System and Signal Processing 14(2), p.181-192.

6. SPLIID, H. A Fast Estimation for the Vector Autoregressive Moving Average Models With Exogenous Variables. Journal of the American Statistical Association, 83 1097-1107. 1983
7. Abdelghanil, M., Gousat, M. y Biolchii, T. (1999). On-line Modal monitoring of Aircraft Structures Under Unknown Excitation. Mechanical System and Signal Processing. 13(6), p.839-853.

8. Cooper, J. E., Desforges, M. J. y Wright, J. R. (1995). Modal Parameter Identification using an Unknown Coloured Random Input. Mechanical System and Signal Processing. 9(6), p.685-695.

9. Huang, C. S. (2001). Structural Identification from Ambient Vibration Measurement Using the Multivariate AR Model. Journal of Sound and Vibration. 241(3), p.337-359.

10.Lee, A. C. y Chen, J. H. (1999). Modal Analysis for Randomly Excited Structural Systems with Unmeasured Input. Journal of Sound and Vibration. 132(1), p.101-113.

11.Shumway, R. H. y Stoffer, D. S. (2000). Time Series Analysis andits Applications. New York. p. 549. Springer-Verlag. 